\title{
Exact Holography of Massive M2-brane Theories and Entangle- ment Entropy
}

\author{
Dongmin Jang ${ }^{1, \star}$, Yoonbai Kim ${ }^{1, \star \star}$, O-Kab Kwon ${ }^{1, \star \star \star}$, and D. D. Tolla ${ }^{1,2, \star \star \star \star}$ \\ ${ }^{1}$ Department of Physics, BK21 Physics Research Division, Institute of Basic Science, Sungkyunkwan Uni- \\ versity, Suwon 440-746, South Korea \\ ${ }^{2}$ University College, Sungkyunkwan University, Suwon 440-746, South Korea
}

\begin{abstract}
We test the gauge/gravity duality between the $\mathcal{N}=6$ mass-deformed ABJM theory with $\mathrm{U}_{k}(N) \times \mathrm{U}_{-k}(N)$ gauge symmetry and the 11-dimensional supergravity on LLM geometries with $S O(4) / \mathbb{Z}_{k} \times S O(4) / \mathbb{Z}_{k}$ isometry. Our analysis is based on the evaluation of vacuum expectation values of chiral primary operators from the supersymmetric vacua of mass-deformed ABJM theory and from the implementation of Kaluza-Klein (KK) holography to the LLM geometries. We focus on the chiral primary operator (CPO) with conformal dimension $\Delta=1$. The non-vanishing vacuum expectation value (vev) implies the breaking of conformal symmetry. In that case, we show that the variation of the holographic entanglement entropy (HEE) from it's value in the CFT, is related to the non-vanishing one-point function due to the relevant deformation as well as the source field. Applying Ryu Takayanagi's HEE conjecture to the 4-dimensional gravity solutions, which are obtained from the KK reduction of the 11-dimensional LLM solutions, we calculate the variation of the HEE. We show how the vev and the value of the source field determine the HEE.
\end{abstract}

\footnotetext{
^e-mail: dongmin@skku.edu

$\star \star$ e-mail: yoonbai@skku.edu

$\star \star \star$ e-mail: okab@skku.edu

$\star \star \star \star$ e-mail: ddtolla@skku.edu
} 


\section{Introduction}

We consider a non conformal quantum field theory and test the gauge/gravity duality away from the conformal fixed point in the large $N$ limit. We start with the $\mathcal{N}=6 \mathrm{U}_{k}(N) \times \mathrm{U}_{-k}(N)$ Aharony-BergmanJafferis-Maldacena (ABJM) theory with Chern-Simons level $k$ [1], as the CFT at the UV fixed point. The ABJM theory allows the supersymmetry preserving mass deformation and the deformed theory (mABJM) [2, 3] has discrete Higgs vacua presented by the Gomis, Rodriguez-Gomez, Van Raamsdonk, Verlinde (GRVV) matrices [3]. It was known that the vacua of the mABJM theory have one-toone correspondence $[4,5]$ with the half BPS Lin-Lunin-Maldacena (LLM) geometries [6, 7] having $\mathrm{SO}(2,1) \times \mathrm{SO}(4) / \mathbb{Z}_{k} \times \mathrm{SO}(4) / \mathbb{Z}_{k}$ isometry in 11-dimensions [5, 8]. Since the mABJM theory is obtained by a relevant deformation from the ABJM theory at the UV fixed point, the dual geometry should be asymptotically $\mathrm{AdS}_{4} \times S^{7} / \mathbb{Z}_{k}$. We test this gauge/gravity duality, by calculating the vacuum expectation values (vevs) of the chiral primary operator with conformal dimension $\Delta=1$ using both sides of the duality.

The novelty of gauge/gravity duality is that, quantities which are difficult to calculate on one side of the duality can easily be obtained on the other side. One such case is the calculation of entanglement entropy in dimension higher than two, which is very difficult to obtain from the quantum field theory. However, Ryu and Takanayagi (RT) suggested a holographic method of entanglement entropy calculation, for $d$-dimensional conformal field theory from the $\mathrm{AdS}_{d+1}$ solution of the dual gravity [9]. In this letter, we extend RT conjecture to non-conformal case and calculate the entanglement entropy for $\mathrm{mABJM}$ from an asymptotically $\mathrm{AdS}_{4}$ solution of 4-dimensional supergravity, which is obtained by the KK reduction of the LLM solutions.

\section{Construction of 4-dimensional Gravity}

A 4-dimensional gravity theory on an asymptotically $\mathrm{AdS}_{4}$ background can be constructed from the KK reduction of 11-dimensional gravity on the LLM geometries. The KK reduction procedure involves, expansion of the 11-dimensional fields in terms of the spherical harmonics on $S^{7}$ and then projecting the 11-dimensional equations of motion on the appropriate spherical harmonic elements. The resulting equations are diagonalized to obtain the equations for infinite towers of gauge invariant $\mathrm{KK}$ modes. In general, this procedure results in the field equations which contain higher derivative terms. In order to absorb the higher derivative terms and write the canonical 4-dimensional equations of motion for those KK modes, we need to introduce some field redefinitions. Such field redefinitions are some times called the KK maps. See [11, 12] for details.

Following the above procedure and considering the small mass parameter expansion, only the equations of motion of few KK modes, which includes the 4-dimensional graviton mode $H_{\mu \nu}$, one scalar mode $\Psi$, and one pseudo scalar mode $T$, are non trivial at quadratic order in the mass parameter. These and all the other 4-dimensional fields are related to the 11-dimensional fields by the KK maps. The equations of motion of those three modes are

$$
\begin{aligned}
& \left(\square-M_{T}^{2}\right) T=0, \quad\left(\square-M_{\Psi}^{2}\right) \Psi=0, \quad \text { with } M_{T}^{2}=M_{\Psi}^{2}=-\frac{8}{L^{2}} \\
& \frac{1}{2}\left(-\square H_{\mu \nu}+\nabla^{\rho} \nabla_{\mu} H_{v \rho}+\nabla^{\rho} \nabla_{\nu} H_{\mu \rho}-\nabla_{\mu} \nabla_{\nu} H\right)+\frac{12}{L^{2}} H_{\mu \nu} \\
& +8 \pi G_{N} A_{T}\left(\nabla_{\mu} T \nabla_{\nu} T+\frac{M_{T}^{2}}{2} g_{\mu \nu}^{(0)} T^{2}\right)+8 \pi G_{N} A_{\Psi}\left(\nabla_{\mu} \Psi \nabla_{\nu} \Psi+\frac{M_{\Psi}^{2}}{2} g_{\mu \nu}^{(0)} \Psi^{2}\right)=0,
\end{aligned}
$$

where $g_{\mu \nu}^{(0)}$ is metric of the pure $\mathrm{AdS}_{4}, L$ is the radius of $S^{7}$ in 11-dimensions, and $G_{N}$ is the Newtion's constant in 4-dimensions. For the detailed definitions of parameters, see [11, 12]. 
Asymptotically $\mathrm{AdS}_{4}$ solutions of the 4-dimensional equations of motion are obtained from the asymptotic expansion of the LLM solutions in 11-dimensions. Performing the expansion we obtain the following results

$$
\begin{aligned}
& H_{i j}=\left[-\frac{\left(L \mu_{0}\right)^{2}}{180}\left(30+\beta_{3}^{2}\right)+O\left(\mu_{0}^{4}\right)\right] \eta_{i j} \quad H_{z z}=-\frac{\left(L \mu_{0}\right)^{2}}{1440}\left(960+29 \beta_{3}^{2}\right)+O\left(\mu_{0}^{4}\right), \\
& \Psi=-24 \beta_{3} \mu_{0} z+O\left(\mu_{0}^{3}\right), \quad T=16 \sqrt{3} \mu_{0} z+O\left(\mu_{0}^{3}\right),
\end{aligned}
$$

where $\mu_{0}$ is the mass parameter of the LLM solutions and $\beta_{3}$ parametrizes all LLM solutions. We have verified that $\Psi$ is dual to CPO of conformal dimension one, while $T$ is dual to a gauge invariant operator of conformal dimension two. Using these 4-dimensional gravity results, we examine the vev of chiral primary operators and holographic entanglement entropy in mABJM theory.

\section{Exact Holography}

In mABJM theory, there is a unique CPO with conformal dimension one $\left(O^{(1)}\right)$ that have a nonvanishing vev $[11,12]$. Following the standard perturbative expansion in mABJM theory, we can calculate the one point function for such CPO and express the result as an expansion in $1 / N$. In the large $N$ limit, only the leading terms which is completely determined by the discrete Higgs vacua of the $\mathrm{mABJM}$ theory is relevant. See $[11,12]$ for details. The result is

$$
\left\langle O^{(1)}\right\rangle_{m}=\frac{k \mu}{4 \sqrt{2} \pi} \sum_{n=0}^{2 N_{\mathrm{B}}+1} n(n+1)\left(N_{n}-N_{n}^{\prime}\right),
$$

where $\left\{N_{n}, N_{n}^{\prime}\right\}$ are the occupation numbers of the discrete Higgs vacua, and $\mu=4 \mu_{0}$ is the mass parameter in the mABJM theory. This result is identified with the coefficient of the asymptotic expansion of the dual scalar field $\Psi$ in (2), by using the one-to-one correspondence between the discrete Higgs vacua and the LLM solutions. Then, the vev is given by

$$
\left\langle O^{(1)}\right\rangle=\frac{N^{\frac{3}{2}} \mu_{0}}{3 \sqrt{2} \pi} \beta_{3} .
$$

\section{Holographic Entanglement Entropy}

According to the RT conjecture, the HEE with a subsystem $A$ on the boundary of $(d+1)$-dimensional AdS geometry is given by $S_{A}=\frac{\operatorname{Min}\left(\gamma_{A}\right)}{4 G_{N}}$, where $G_{N}$ is the Newton constant in the $(d+1)$-dimensional gravity theory and $\gamma_{A}$ is an area of the surface stretched to the bulk direction, which has the same boundary with the subsystem $A$ [9]. The 4-dimensional metric obtained in section 2 is a perturbation of pure $\mathrm{AdS}_{4}, g_{\mu \nu}=g_{\mu \nu}^{(0)}+H_{\mu \nu}$. Then the induced metric is given by

$$
\tilde{g}_{i j}=\frac{\partial x^{\mu}}{\partial \sigma^{i}} \frac{\partial x^{v}}{\partial \sigma^{j}} g_{\mu v}=\frac{\partial x^{\mu}}{\partial \sigma^{i}} \frac{\partial x^{v}}{\partial \sigma^{j}}\left(g_{\mu \nu}+H_{\mu \nu}\right)=\tilde{g}_{i j}+\tilde{H}_{i j}
$$

The deviation $\tilde{H}_{i j}$ of the induced metric from its $\mathrm{AdS}_{4}$ value results in the variation of the area for the surface whose boundary is a disk of radius $l$,

$$
\delta \gamma_{A}=\frac{1}{2} \int d^{2} \sigma \sqrt{\operatorname{det} \tilde{g}_{i j}} \tilde{g}^{i j} \tilde{H}_{i j}=\pi \int_{0}^{l} d \rho \sqrt{\operatorname{det} \tilde{g}_{i j}} \tilde{g}^{i j} \tilde{H}_{i j}
$$


Inserting the results in (2) into (5), we obtain the expansions of $\tilde{H}_{i j}$ up to $\mu_{0}^{2}$-order. Using those expansions in (6), we obtain the variation of the minimum area,

$$
\delta \gamma_{A}=-\frac{\pi L^{2} \mu_{0}^{2}}{1440} \int_{0}^{l} d \rho \frac{\rho}{\sqrt{1+z^{\prime 2}}}\left[\left(1200+37 \beta_{3}^{2}\right) z^{\prime 2}+16\left(30+\beta_{3}^{2}\right)\right]=-\frac{\pi L^{2}\left(\mu_{0} l\right)^{2}}{48}\left(32+\beta_{3}^{2}\right) .
$$

Therefore, the HEE up to $\mu_{0}^{2}$-order is given by

$$
S_{A}=S_{A}^{(0)}+\delta S_{A}=\frac{\pi L^{2}}{8 G_{N}}\left[\frac{l}{\epsilon}-1-\frac{4}{3}\left(1+\frac{\beta_{3}^{2}}{32}\right)\left(\mu_{0} l\right)^{2}\right] .
$$

We see that the solutions of the 4-dimensional metric (2) for all possible droplets exactly reproduce the HEE which was calculated from the 11-dimensional LLM solutions [10].

Since the metric deformation in (2) is due to the presence of the matter fields $\Psi$ and $T$, we conclude that the $\beta_{3}$-term in $\delta S_{A}$ in (7) is originated from the vevs of the CPO $O^{(1)}$, which is dual to the scalar field $\Psi$, while the constant term in $\delta S_{A}$ is originated from the source of a gauge invariant operator $\tilde{O}^{(2)}$, which is dual to the pseudo scalar field $T$. Therefore, we see the HEE up to $\mu_{0}^{2}$-order in the large $N$ limit is the function of the source and the vevs, $\delta S_{A}=\delta S_{A}\left(J_{\tilde{O}^{(2)}},\left\langle O^{(1)}\right\rangle\right)$, where $J_{\tilde{O}^{(2)}}$ denotes the source of $\tilde{O}^{(2)}$.

\section{Conclusion}

In this letter, we carried out the KK reduction and the holographic renormalization procedure for the mABJM theory and the LLM geometry in 11-dimensional supergravity. By calculating the vevs of CPO with $\Delta=1$ in field theory side and the corresponding asymptotic coefficients in gravity side, we found a supporting evidence for an exact gauge/gravity duality with $k=1$ in the large $N$ limit. We could test the duality since discrete Higgs vacua exist in the mABJM theory and they correspond one-to-one with the LLM geometries. We also extended the exact holographic relation to the case of any $k$ for LLM geometries represented by rectangular-shaped Young-diagrams.

It seems that the Higgs vacua of the mABJM theory are parametrized by the vevs of CPOs and those are nonrenormalizable due to the high supersymmetry. This is similar to the case of the Coulomb branch in large $N$ limit in $\mathcal{N}=4$ super Yang-Mills theory [13,14]. Though our quantitative results for the gauge/gravity correspondence involve infinite examples, we need to accumulate more analytic evidences for CPOs with $\Delta(\geq 2)$ and $k(\geq 1)$ to define supersymmetric vacua. One should also test the dictionary of the gauge/gravity duality for one point functions of vector and tensor fields. For instance, it is important to verify that one point functions of the energy-momentum tensor vanish for all possible supersymmetric vacua, since the mABJM theory is a supersymmetric theory. We leave these issues for future study.

\section{Acknowledgements}

This work was supported by the National Research Foundation of Korea(NRF) grant with the grant number NRF-2016R1D1A1B03931090 (Y.K.), NRF-2017R1D1A1A09000951 (O.K.), NRF2017R1D1A1B03032523 (D.T.).

\section{References}

[1] O. Aharony, O. Bergman, D. L. Jafferis and J. Maldacena, JHEP 0810, 091 (2008). 
[2] K. Hosomichi, K. M. Lee, S. Lee, S. Lee and J. Park, JHEP 0809, 002 (2008).

[3] J. Gomis, D. Rodriguez-Gomez, M. Van Raamsdonk and H. Verlinde, JHEP 0809, 113 (2008).

[4] H. C. Kim and S. Kim, Nucl. Phys. B 839, 96 (2010).

[5] S. Cheon, H. C. Kim and S. Kim, arXiv:1101.1101 [hep-th].

[6] I. Bena and N. P. Warner, JHEP 0412, 021 (2004).

[7] H. Lin, O. Lunin and J. M. Maldacena, JHEP 0410, 025 (2004).

[8] R. Auzzi and S. P. Kumar, JHEP 0910, 071 (2009).

[9] S. Ryu and T. Takayanagi, Phys. Rev. Lett. 96, 181602 (2006); JHEP 0608, 045 (2006).

[10] K. K. Kim, O. K. Kwon, C. Park and H. Shin, Phys. Rev. D 90, no. 4, 046006 (2014); Phys. Rev.

D 90, no. 12, 126003 (2014); C. Kim, K. K. Kim and O. K. Kwon, Phys. Lett. B 759, 395 (2016).

[11] D. Jang, Y. Kim, O. K. Kwon and D. D. Tolla, Eur. Phys. J. C 77, no. 5, 342 (2017); JHEP 1704, 104 (2017).

[12] D. Jang, Y. Kim, O. K. Kwon and D. D. Tolla, in preparation.

[13] K. Skenderis and M. Taylor, JHEP 0605, 057 (2006).

[14] K. Skenderis and M. Taylor, JHEP 0608, 001 (2006). 